\title{
Nanoporous PMMA foams with templated pore size obtained by localized in situ synthesis of nanoparticles and $\mathrm{CO}_{2}$ foaming
}

\author{
Javier Pinto ${ }^{\text {a, }}{ }^{*}$, Davide Morselli a, Victoria Bernardo ${ }^{b}$, Belen Notario ${ }^{b}$, Despina Fragouli a , \\ Miguel Angel Rodriguez-Perez ${ }^{\mathrm{b}}$, Athanassia Athanassiou ${ }^{\text {a, }}{ }^{* *}$ \\ a Smart Materials - Nanophysics Department, Istituto Italiano di Tecnologia (IIT), Genova, Italy \\ ${ }^{\mathrm{b}}$ Cellular Materials Laboratory (CellMat), Condensed Matter Physics Department, University of Valladolid, Valladolid, Spain
}

\section{A R T I C L E I N F O}

\section{Article history:}

Received 10 April 2017

Received in revised form

31 May 2017

Accepted 24 July 2017

Available online 26 July 2017

\section{Keywords:}

Polymer foam

Heterogeneous nucleation

ZnO nanoparticles

\begin{abstract}
A B S T R A C T
Polymer foams with controlled and templated pore size have been obtained for the first time by $\mathrm{CO}_{2}$ gas dissolution foaming from poly(methyl methacrylate) (PMMA) films. This kind of materials, with a variable porous structure, mimic some high-performance natural materials and could present significant interest in many applications. However, up to now their controlled fabrication has not been successfully achieved. Herein, we present a method to achieve a fine control in the production of such materials. Thermal in situ synthesis of $\mathrm{ZnO}$ nanoparticles from $\mathrm{Zn}(\mathrm{OAc})_{2}$ was proposed to obtain PMMA nanocomposites, in which the $\mathrm{ZnO}$ nanoparticles induce heterogeneous nucleation that leads to formation of pores with size below the micron, upon $\mathrm{CO}_{2}$ foaming. Starting from templated solid PMMA samples with well-differentiated regions, presenting or not $\mathrm{ZnO}$ nanoparticles, it was possible to obtain PMMA-based foams with well-defined areas of different pore sizes.
\end{abstract}

(c) 2017 Elsevier Ltd. All rights reserved.

\section{Introduction}

Polymer foams are lightweight materials widely employed in several industries such as the automobile, aerospace, transportation, packaging, and construction. In addition to the weight reduction, polymer foams can present interesting thermal/acoustic insulation, energy absorption, flexibility, and excellent mechanical properties [1]. Therefore, the increasing market demand for materials with higher performances and lower weights promotes the continuous research on the development of improved polymer foams. For instance, polymer foams with improved mechanical properties could allow significant weight reductions in transportation, decreasing energy consumption and $\mathrm{CO}_{2}$ emissions [2]; whereas an enhanced thermal insulation can provide similar advantages on the households heating, supporting a sustainable development [3].

Since the 1980s, with the development of microporous polymer foams at Massachusetts Institute of Technology [1], it has been

\footnotetext{
* Corresponding author.

** Corresponding author

E-mail addresses: jpintosanz@gmail.com (J. Pinto), Athanassia.Athanassiou@iit.it (A. Athanassiou).
}

clearly demonstrated that the pore size plays a key role on the macroscopic physical properties of polymer foams. This concept has been further supported in the last years with the development of nanoporous polymer foams, which have shown a wide range of improved or even unexpected physical properties due to their pore size well below the micron [3-13]. Nanoporous polyetherimide (PEI) foams have shown increased strain to failure and tensile toughness compared to microporous PEI foams [6]; whereas nanoporous poly(methyl methacrylate)(PMMA) foams have shown higher modulus of elasticity, higher impact strength, and improved hardness with respect to microporous PMMA foams [7]. Furthermore, these materials have shown increased thermal insulation performances due to Knudsen effect [3,8,9]. This effect happens when the pore size values become comparable to the mean free path of the air molecules (about $70 \mathrm{~nm}$ under standard conditions), decreasing significantly the air thermal conductivity inside the pores [14]. Finally, previous works also found a modification of the dielectric and acoustic properties of PMMA foams when the pore size decreases from the micro to the nanometric range [10,11], as well as an increase of the polymer matrix $\mathrm{Tg}$ associated to the molecular confinement of the PMMA chains in the thin pore walls (with a thickness below 100-150 nm) [12,13].

The experimental confirmation of these advantages of the nanoporous polymer foams have recently increased the academic 
and industrial interest in the production of these materials. In the last years, gas dissolution foaming using $\mathrm{CO}_{2}$ as physical blowing agent has become one of the preferred routes for the fabrication of nanoporous polymer foams [4,5,8,9,12,15-39]. This technique presents several advantages, such as its environmental friendliness, absence of chemical residues, and easy processability $[4,5,8,9]$. However, the production of nanoporous foams using this technique requires reduced pore coalescence and coarsening, as well as very high pore nucleation ratios [4,40-43]. This can be achieved by employing amorphous polymers with high glass transition temperature, such as PEI or PMMA, whose large viscosity prevents pore coalescence if the foaming step is conducted in the right processing window [31,44]. On the top, very high pore nucleation ratios require polymers with high $\mathrm{CO}_{2}$ affinity (e.g. PMMA) and the use of extreme processing conditions (e.g. very high gas saturation pressure and/or very high pressure drop rate) (homogeneous nucleation) $[12,18,21,42]$ or the addition of nucleation agents to the polymer (heterogeneous nucleation) [15,16,18,19,45-48]. From an industrial point of view the use of nucleation agents is preferable, as they allow production of nanoporous foams using mild processing conditions (i.e. increasing the safety of the procedure and decreasing the costs) [18].

Focusing on PMMA, which according to the literature is one of the most promising polymers to obtain nanocellular foams [12,15-18,25,28,34,35,46], two kinds of nucleation agents have been successfully employed so far: nanostructured block copolymers and nanoparticles. Poly(methyl methacrylate)- $b$-poly(butyl acrylate)- $b$-poly(methyl methacrylate) (MAM) triblock copolymers have been employed by Pinto et al. [18] and Forest et al. [15] to obtain nanoporous foams with pore sizes of about $100-200 \mathrm{~nm}$ and relative densities of about $0.4-0.5$. In these works, blends of PMMA and MAM showed a well-defined nanostructuration, which controls the pore nucleation independently of the foaming parameters, allowing to obtain one pore by each initial nanodomain and achieving a pore nucleation density about $4 \times 10^{14}$ pores per cubic centimetre of the solid precursor [18]. Whereas Costeux and Zhu [16] introduced silica nanoparticles in a PMMA matrix to promote the nucleation, obtaining similar pore sizes with the previous approach (about $100 \mathrm{~nm}$ ) but a significantly lower relative density of 0.15 due to a remarkable higher pore nucleation (over $10^{16}$ pores per cubic centimetre of the solid precursor). Therefore, the use of nanoparticles (NPs) as nucleation agents in polymers seems to be a suitable approach to provide pore nucleation densities high enough to obtain low density nanocellular foams. However, in the production of nanocomposite materials, the NPs dispersion is the most critical parameter to control [49]. It is well known that nanoparticles arising from flame pyrolysis or wet chemistry cannot be easily re-dispersed in polymeric matrices, by simply using the conventional mixing methods, due to their strong tendency to form micrometric agglomerates [50].

For overcoming such drawback, an alternative and innovative method is the so-called "in situ synthesis of NPs" in which the desired filler is directly synthesized in a hosting polymeric solid matrix, therefore preventing the NPs aggregation [50]. Typically, a previously dispersed precursor is converted in the desired NPs by a simple thermal treatment [51,52] or by laser irradiation [53-55] resulting in the formation of particles homogeneously dispersed in the polymeric matrix. In the literature only a few studies on this approach are reported for a specific application, for instance Beek et al. and Reynolds et al. describe the thermally induced formation of $\mathrm{ZnO}$ [56-59] and CdS [60,61] NPs, respectively, in conductive polymers to apply them in hybrid polymer solar cells.

Despite this approach has not been frequently reported in literature, it may ensure important enhancements in several technological applications where polymer-based nanocomposites are employed such as the production of polymer foams. Herein, we reported the use of the in situ synthesis of NPs as a method to obtain suitable PMMA-based nanocomposites for the production of nanoporous foams via $\mathrm{CO}_{2}$ foaming. Firstly, we demonstrated that the thermal in situ synthesis of ZnO nanoparticles, from PMMA/ $\mathrm{Zn}(\mathrm{OAc})_{2}$ samples, allows the decrease of the pore size from $11 \mu \mathrm{m}$ to $500 \mathrm{~nm}$ in PMMA-based samples foamed at $20 \mathrm{MPa}$ and $60^{\circ} \mathrm{C}$.

Then, taking advantage of this procedure we studied the possibility to obtain foams with a controlled and templated porous structure, presenting distinct nanoporous and microporous areas that follow a pre-existing NPs template obtained by a selective in situ synthesis of the NPs. Polymer foams with a controlled pore size gradient have been proposed as a way to reach improved acoustic insulation [62] or electromagnetic interference shielding [63], as well as to mimic high-performance biological structures (e.g. bones) [64]. A few attempts to obtain this kind of structures using $\mathrm{CO}_{2}$ foaming can be found in the literature $[64,65]$. Yu et al. [64] obtained PMMA, polystyrene (PS), and poly(lactic acid) (PLA) foams with a pore size gradient induced by the presence of a metallic film on one of the faces of the polymer precursor; obtaining the best results with PLA, which presented a pore size gradient between 13 and $40 \mu \mathrm{m}$. Ngo et al. [65] employed a specific foaming setup, including a foaming vessel in which PMMA long strips $(25 \mathrm{~cm})$ were foamed under a temperature gradient, obtained a heterogeneous expansion of the PMMA strip which leads to different porosities and pore sizes, but always with cell sizes in the microporous range. In this work PMMA/Zn $(\mathrm{OAc})_{2}$ samples were subjected to heterogeneous thermal treatment where one half was heated up to $100{ }^{\circ} \mathrm{C}$ while the other half was kept at $0{ }^{\circ} \mathrm{C}$. After this treatment the samples presented a well-defined template with half of them being composed by PMMA/ZnO NPs and the other half by $\mathrm{PMMA} / \mathrm{Zn}(\mathrm{OAc})_{2}$. Finally, PMMA-based foams with controlled tunable pore size between the nanometric and micrometric range were obtained by $\mathrm{CO}_{2}$ foaming. As far as we know, this is the first time that the in situ synthesis of NPs has been employed for the production of nanoporous foams, as well as the first time that polymer foams with controlled and templated pore sizes, simultaneously in the micro and nanometric ranges, have been obtained.

\section{Experimental section}

\subsection{Materials}

Poly(methyl methacrylate) (PMMA, average molecular weight $~ 350 \mathrm{kDa})$, Zinc acetate dihydrate $\left(\mathrm{Zn}(\mathrm{OAc})_{2}, 99,999 \%\right), 2-$ propanol (LC-MS Chromasolv ${ }^{\circledR}$ ), toluene (Chromasolv ${ }^{\circledR}$ Plus), hydrochloridric acid $\left(\mathrm{HCl}, 37 \%\right.$, ACS reagent) and nitric acid $\left(\mathrm{HNO}_{3}\right.$, $70 \%$, ACS reagent) were purchased by Sigma Aldrich (Milan, Italy). All reported chemicals were high-purity reagents and they were used as received without any further purification. Medical grade $\mathrm{CO}_{2}$ (99.9\% purity) was used as blowing agent.

\subsection{Preparation of PMMA/ZnO nanocomposite}

$200 \mathrm{~g}$ of PMMA were dissolved in $20 \mathrm{~mL}$ of toluene overnight by heating at $60^{\circ} \mathrm{C}$ on a hot plate. Afterwards, $5.4 \mathrm{mg}$ of $\mathrm{Zn}(\mathrm{OAc})_{2}$ were dissolved in $1.2 \mathrm{~mL}$ of 2-propanol by ultrasonic bath (LBS 2, Falc) for $30 \mathrm{~min}$ at $59 \mathrm{kHz}$, and subsequently by stirring for $3 \mathrm{~h}$ at room temperature and $1 \mathrm{~h}$ at $45^{\circ} \mathrm{C}$.

The $\mathrm{Zn}(\mathrm{OAc})_{2}$ solution was then added to PMMA solution and left under vigorous stirring for $15 \mathrm{~min}$ at room temperature. The soobtained mixture was sonicated by probe sonicator (Vibra Cel, Sonics) using three pulses (intensity $20 \mathrm{kHz}, 15 \mathrm{~s}$ each and pulse amplitude of $50 \%) .5 \mathrm{~mL}$ of the final mixture were cast in a circular Teflon mould ( $38 \mathrm{~mm}$ diameter $\times 5.5 \mathrm{~mm}$ depth) and left to dry 
under an aspiration hood for $24 \mathrm{~h}$. Finally, the dry films were detached from the mould and further dried for $6 \mathrm{~h}$ under dynamic vacuum in order to completely remove the possible entrapped solvent.

PMMA/Zn(OAc) $)_{2}$ films (precursor content is about $2.7 \mathrm{wt} \%$ ) were annealed in an oven at $110{ }^{\circ} \mathrm{C}$ for $14 \mathrm{~h}$ in order to thermally activate the in situ synthesis reaction for obtaining ZnO NPs (about $1 \mathrm{wt} \%$ with respect to the PMMA, assuming a full conversion of the precursor [55]). This amount of precursor/ZnO NPs was determined as optimum for this application, lower amounts do not provide pore nucleation densities high enough to obtain nanoporous foams, while higher amounts presented an inappropriate NPs dispersion.

Neat PMMA films were also prepared following the same production route (i.e. solution in toluene and then casted in the Teflon mould) for comparison purposes.

\subsection{Heterogeneous thermal treatment of $P M M A / Z n(O A c)_{2}$ films}

PMMA and PMMA/Zn(OAc) $)_{2}$ film samples with $30 \mathrm{~mm}$ length, $4 \mathrm{~mm}$ width, and $30 \mu \mathrm{m}$ thickness were heterogeneously annealed using a setup in which half the sample was in contact with a heating tape $\left(100{ }^{\circ} \mathrm{C}\right)$ and the other half was in contact with a cold source (about $0^{\circ} \mathrm{C}$ ) for $14 \mathrm{~h}$ (details of this setup can be found in the Supplementary Information).

\subsection{Foaming of PMMA-based films}

Foaming experiments were carried out in a high pressure vessel (model PARR 4681, Parr Instrument Company, USA), with a capacity of $1 \mathrm{~L}$ and capable of operating simultaneously at maximum temperature of $350{ }^{\circ} \mathrm{C}$ and maximum pressure of $41 \mathrm{MPa}$. The vessel is equipped with an accurate pressure pump controller (model SFT10) provided by Supercritical Fluid Technologies Inc. (USA), and controlled automatically to keep the temperature and pressure at the desired values. The foaming production route followed using this system was the so-called solid state foaming [33], composed by three stages. In the first stage the polymer samples are introduced into the pressure vessel under a controlled $\mathrm{CO}_{2}$ high pressure and temperature up to saturation. Then, the pressure is released and the samples are extracted from the vessel, and finally the foaming of the samples is induced by immersion in a thermal bath to heat the samples over the effective glass transition temperature $\left(T_{g, e f f}\right)$ of the polymer $/ \mathrm{CO}_{2}$ system.

Saturation parameters have been chosen as the minimum required to allow a clear foaming of PMMA, PMMA/Zn(OAc $)_{2}$, and PMMA/ZnO films. With this aim saturation pressure was fixed at $20 \mathrm{MPa}$ and saturation temperature at $60{ }^{\circ} \mathrm{C}$, being the saturation time $24 \mathrm{~h}$ for all the experiments. It was found that lower pressures or temperatures do not allow the foaming of some of the materials under study. On the contrary, higher pressures or temperatures allow the foaming but introduce undesired effects. Higher pressures induce a very high homogeneous nucleation which hinder the effect of the heterogeneous nucleation induced by the $\mathrm{ZnO}$ nanoparticles; whereas higher temperatures result in pore coalescence and pore sizes in the micrometre range for all the samples. Finally, the foaming stage was carried out in a water bath at room temperature (RT) for $3 \mathrm{~min}$. It should be noticed that the $T_{g, e f f}$ of the $\mathrm{PMMA} / \mathrm{CO}_{2}$ system can reach values even below room temperature (RT), and therefore the foaming process could start just after the pressure release without need of further heating [44], but this foaming stage was introduced to ensure the same thermal evolution and foaming conditions for all the samples. In addition, it is well-known that the gas dissolution foaming of thin polymer films require to restrict the gas diffusion through the faces of the films [23]. Therefore, the foaming procedure of the PMMA-based films presented in this work was carried out using a specific mould that restricts this diffusion and allows the simultaneous foaming of several samples with $30 \mathrm{~mm}$ length, $4 \mathrm{~mm}$ width, and $30 \mu \mathrm{m}$ thickness (see details in the Supplementary Information).

\subsection{Characterization techniques}

Absorption spectra of the free-standing thermal treated films were recorded on Varian Cary 6000i UV-visible-NIR spectrophotometer (UV-vis) in double beam configuration using PMMA/ $\mathrm{Zn}(\mathrm{OAc})_{2}$ (about $2.7 \mathrm{wt} \%$ ) as a reference sample. The films thickness (approximately $30 \mu \mathrm{m}$ ) was measured using a micrometre (Coolant Proof Micrometre, Mitutoyo) in order to normalize the absorbance, taking into account the optical path. The normalized optical absorption, at about $271 \mathrm{~nm}$, was then plotted as a function of the heating time in order to obtain a kinetic curve of the NPs formation process.

In addition, the presence of the $\mathrm{Zn}(\mathrm{OAc})_{2}$ or $\mathrm{ZnO}$ NPs in the PMMA matrix, and therefore the completion of the synthesis procedure, was further confirmed by X-Ray diffraction (XRD) measurements using a PANalytical Empyrean X-ray diffractometer equipped with a $1.8 \mathrm{~kW} \mathrm{CuK} \alpha$ ceramic X-ray tube $\left(1.1 .5418{ }^{\circ} \mathrm{A}\right)$, PIXcel [3D] $2 \times 2 \mathrm{~mm}^{2}$ area detector and operating at $45 \mathrm{kV}$ and $40 \mathrm{~mA}$. The reflections were collected at room temperature using parallel-beam geometry and symmetric reflection mode, in the range $25-70^{\circ} 2 \theta$ using a step time of $500 \mathrm{~s}$ and a step size of $0.1^{\circ}$, repeating the measurement four times in order to reduce the signal noise. XRD results were analysed using HighScore 4.1 software (PANalytical).

Morphology and dimensions of the primary particles were investigated by a JEOL JEM-1011 transmission electron microscopy (TEM) equipped with a tungsten thermionic electron source, operating at $100 \mathrm{kV}$. About $0.005 \mathrm{~g}$ of the nanocomposite, was dissolved in $1 \mathrm{~mL}$ toluene and left stirring overnight. The obtained solution was sonicated for $1 \mathrm{~h}$, and then a small drop $(5 \mu \mathrm{L})$ was placed on a copper grid (300 Mesh Cu Carbon only) followed by drying at room temperature. The obtained TEM images were analysed and processed (about 50 NPs were measured) by FIJI/Image open-source software [66] in order to evaluate the particles' size distributions.

The solid and porous samples' morphology was investigated by a JEOL JSM 7500FA high resolution scanning electron microscope (HR-SEM) equipped with a cold field emission gun, applying an accelerating voltage of $10 \mathrm{kV}$ and a chamber pressure of $9.6 \times 10^{-5} \mathrm{~Pa}$. The cross-sections were prepared by fracturing the specimens in liquid nitrogen and the so-obtained cross-sections were coated with $10 \mathrm{~nm}$ thick carbon layer by a carbon coater (Emitech K950X high vacuum turbo system, Quorum Technologies Ltd) in order to impart electrical conductivity. Average pore size $\left(\varphi_{3 D}\right)$, porosity $\left(\mathrm{V}_{\mathrm{f}}\right)$, and pore nucleation density $\left(\mathrm{N}_{\mathrm{o}}\right)$ (number of pore nuclei per $\mathrm{cm}^{3}$ of the solid precursor) were obtained from the HR-SEM micrographs using a specific software based on FIJI/Image $[66,67]$.

\section{Results and discussion}

\subsection{In situ synthesized ZnO NPS}

The kinetics of the ZnO NPs formation within the PMMA matrix was followed by monitoring the UV-visible absorption spectra of the samples at different times of annealing at $110{ }^{\circ} \mathrm{C}$ (Fig. 1a). In particular, the representative kinetics curve presented in Fig. 1b is extrapolated by plotting the absorption intensity at $271 \mathrm{~nm}$. This peak is typically attributed to the exciton transition of the small ZnO clusters $[61,68,69]$. As shown in Fig. 1a, already after $1 \mathrm{~h}$ the 

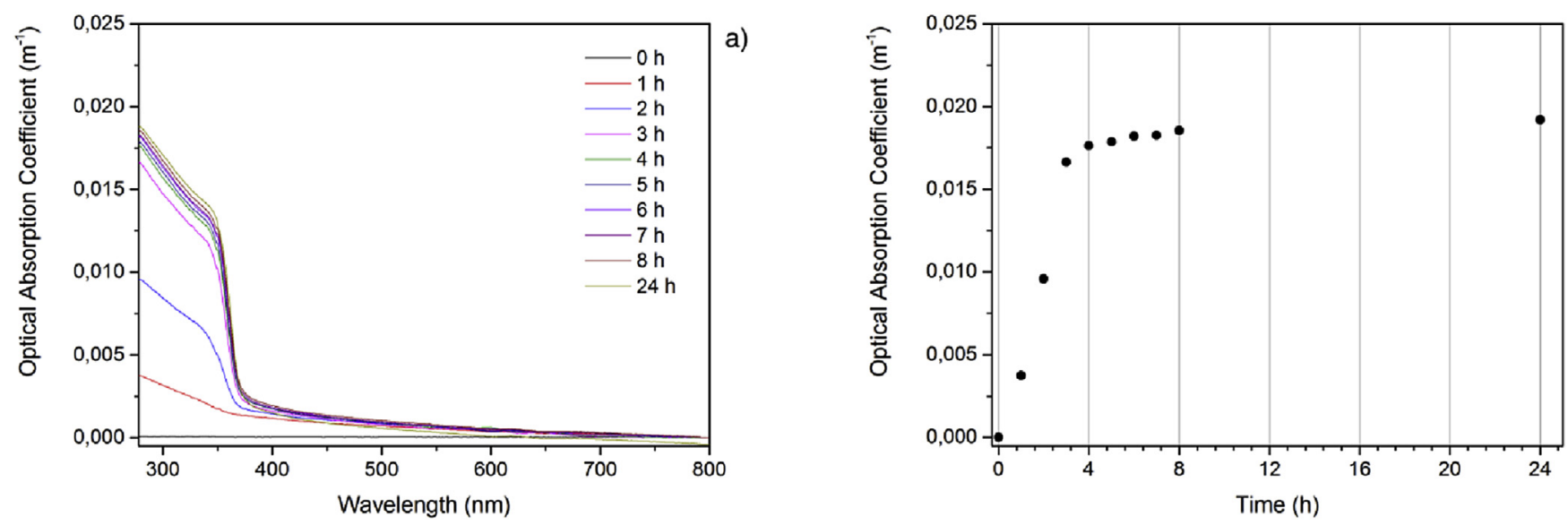

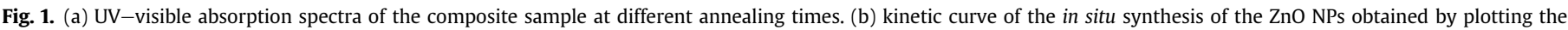
optical absorption at $271 \mathrm{~nm}$ as a function of treatment time.

characteristic absorption band of ZnO NPs arises from the background in the near UV region. Increasing the time of the thermal treatment, the intensity of the absorption band increases linearly up to $3 \mathrm{~h}$, as also clearly shown in the curve reported in Fig. 1b. After this initial increase, the slope of the kinetic curve decreases, and after $8 \mathrm{~h}$ of annealing no significant increments in the absorption of the formed NPs are observed. For this reason $14 \mathrm{~h}$ were chosen as thermal treatment time.

The formation of the ZnO NPs was further confirmed by the XRD measurements presented in Fig. 2. As expected the neat PMMA does not show any reflection peaks, but only a wide band typical of the amorphous material. Before the thermal treatment the composite sample clearly shows only reflections attributed to $\mathrm{Zn}(\mathrm{OAc})_{2}$ (JCPDS file 00-056-0569), whereas after the thermal treatment that reflections disappear and peaks attributed to the presence of the $\mathrm{ZnO}$ NPs arise from the background, demonstrating that the thermal decomposition of the precursor occurred, leading to the formation of ZnO NPs characterized by hexagonal crystalline structure, typical of Zincite phase (JCPDS file 01-079-0206).

Before starting the thermal treatment, the PMMA/Zn(OAc) films present a morphologically homogeneous cross-section (Fig. 3a and b) characterized by the presence of sheet-like submicrometric aggregates representative of $\mathrm{Zn}(\mathrm{OAc})_{2}$, as shown by the TEM image in Fig. 3c. However, after a thermal treatment of $8 \mathrm{~h}$, well-defined ZnO NPs homogeneously grow throughout the polymeric films as depicted in Fig. 4a. Increasing the thermal treatment time to $14 \mathrm{~h}$ an increase of the quantity of NPs is observed as shown in Fig. 4b. Therefore, it was decided to use a $14 \mathrm{~h}$ thermal annealing for the production of the PMMA/ZnO films employed in the foaming experiments.

The morphology, composition and dimensions of NPs were further investigated by the TEM (Fig. $4 \mathrm{c}$ and further images in

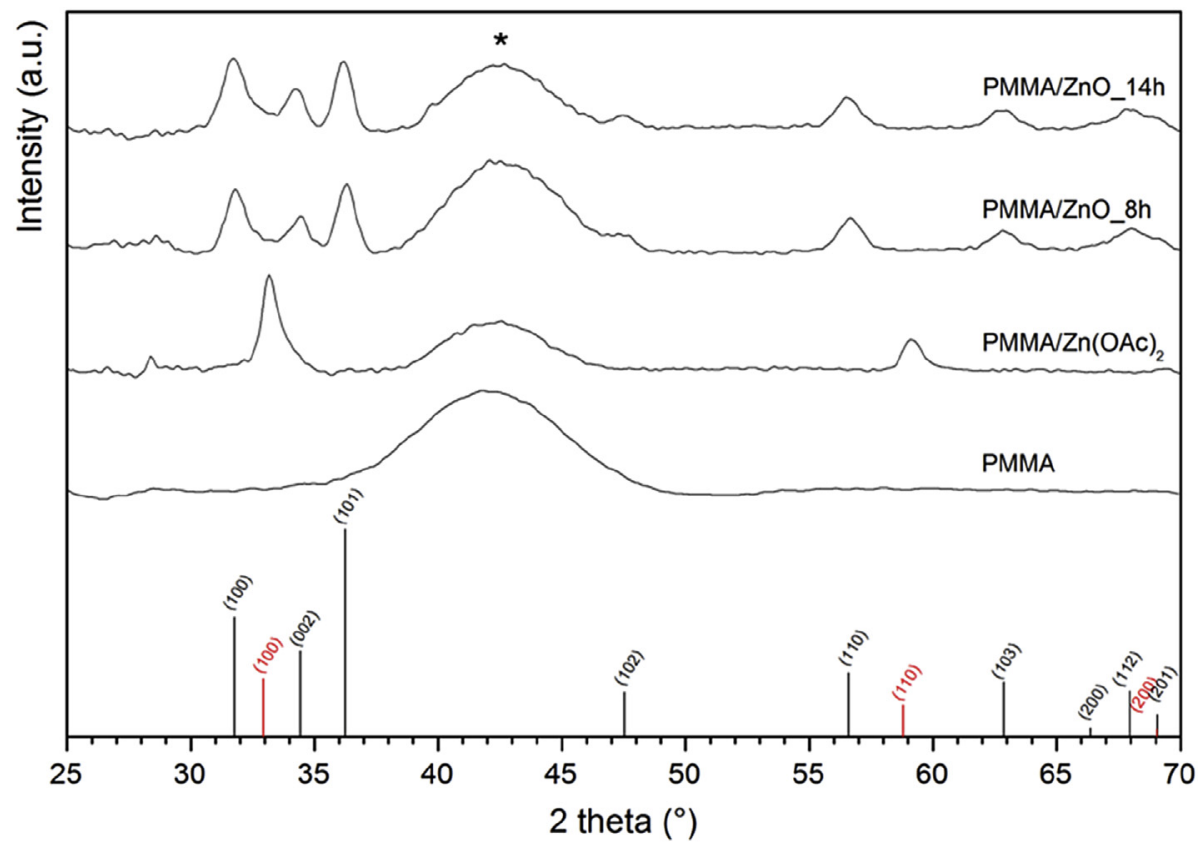

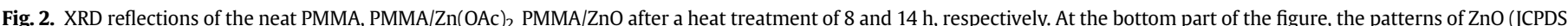

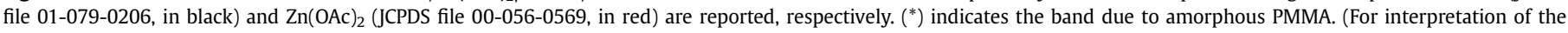
references to colour in this figure legend, the reader is referred to the web version of this article.) 

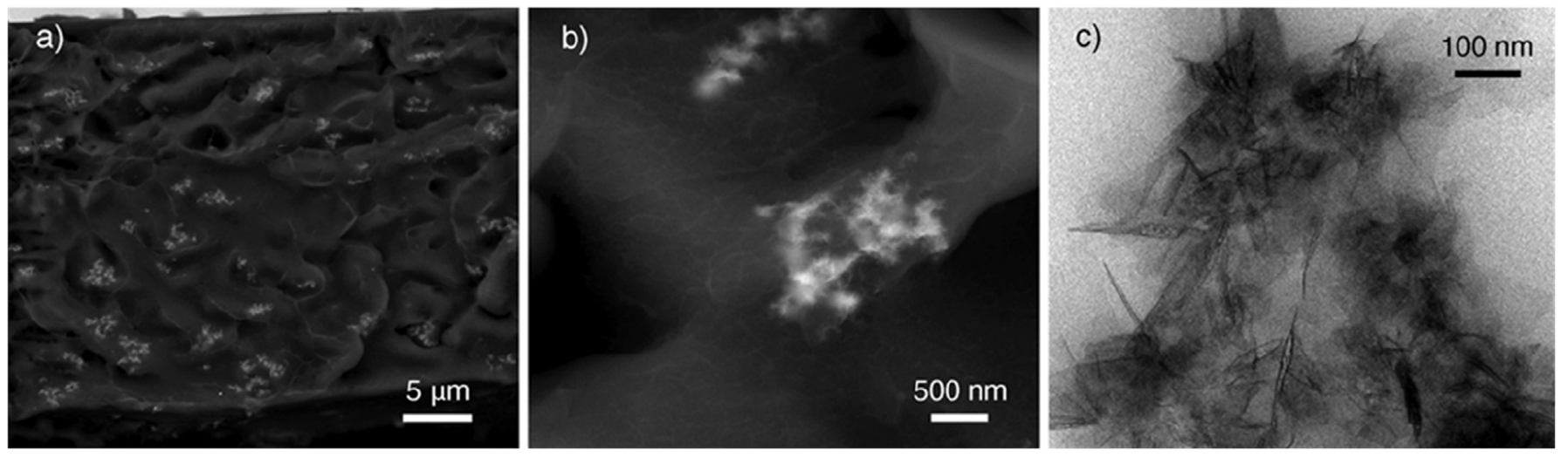

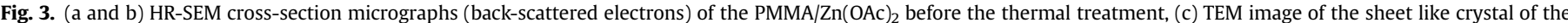
$\mathrm{Zn}(\mathrm{OAc})_{2}$.
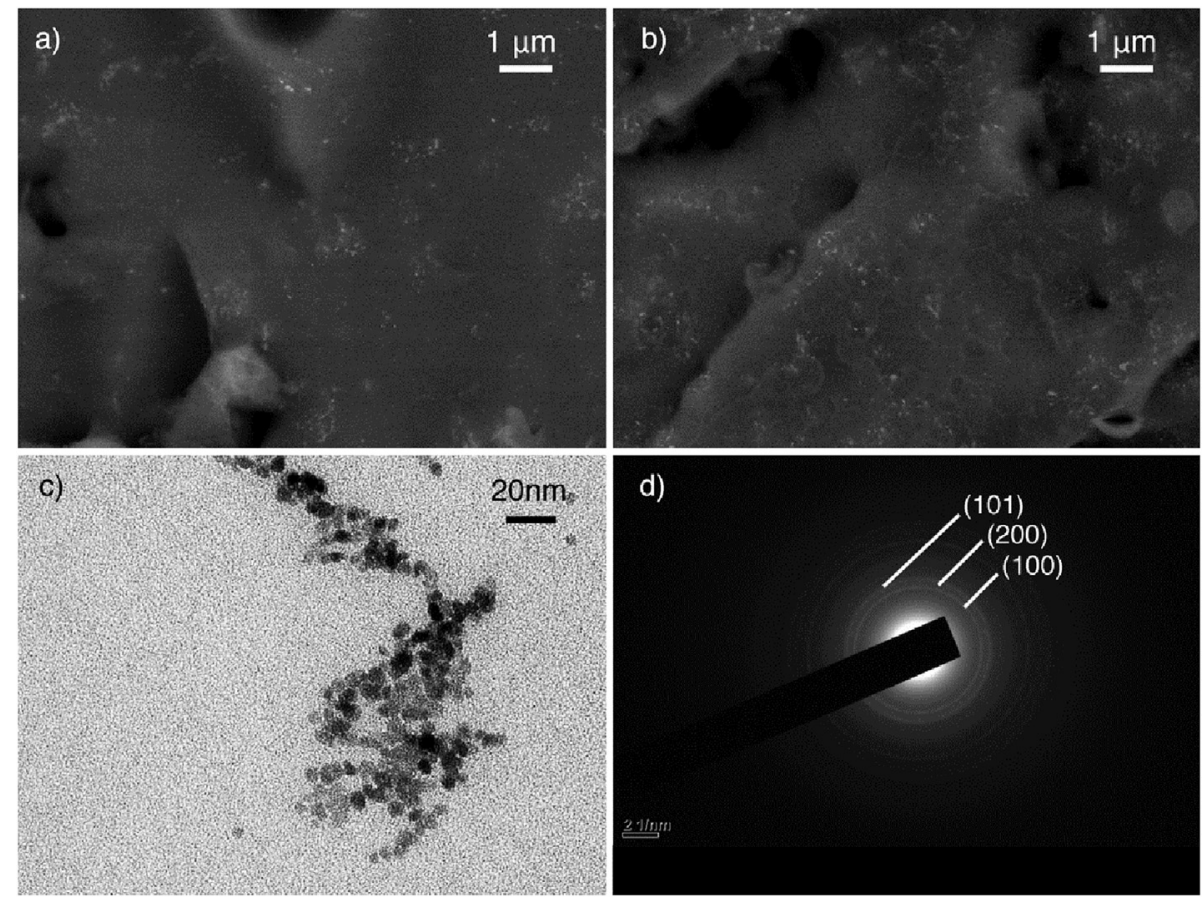

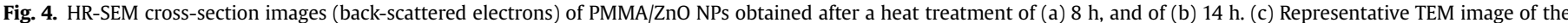
ZnO NPs and (d) electron diffraction patterns of the ZnO NPs observed by TEM.
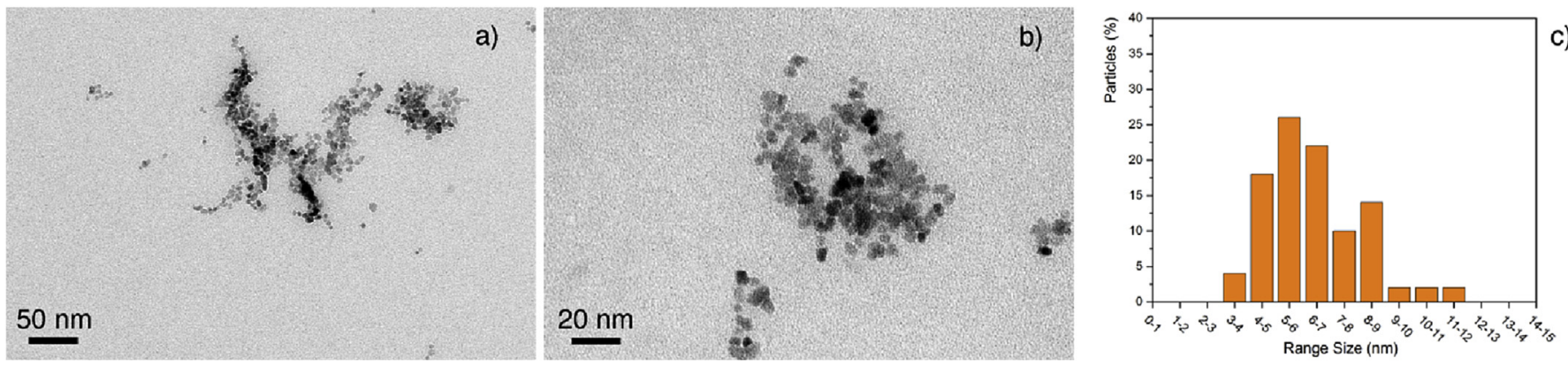

Fig. 5. ( $\mathrm{a}$ and b) TEM micrographs of the in situ synthesized nanoparticles, (c) related particles size distribution.

Fig. 5a and b) that confirmed the rather spherical uniform shape of the particles, while the electron diffraction patterns, displayed in Fig. 4d, confirm that in situ synthesized NPs are composed by crystalline $\mathrm{ZnO}$, in good agreement with $\mathrm{XRD}$ results reported in Fig. 2. Moreover, the primary particles have average diameter of $6.5 \pm 1.2 \mathrm{~nm}$ as shown in the particles size distribution in Fig. 5c. 


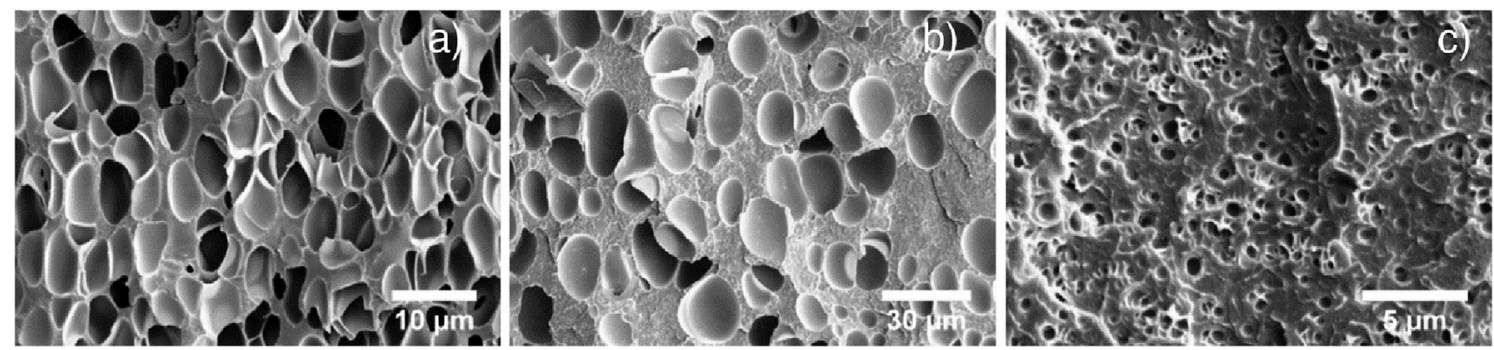

Fig. 6. HR-SEM micrographs of (a) PMMA, (b) PMMA/Zn(OAc) ${ }_{2}$, and (c) PMMA/ZnO NPs foamed films.

\subsection{Foaming of PMMA, PMMA/Zn(OAc) $)_{2}$, and $P M M A / Z n O ~ N P s$ films}

Films of neat PMMA, PMMA/Zn(OAc) $)_{2}$, and PMMA/ZnO NPs (after $14 \mathrm{~h}$ thermal annealing) were subjected to the solid-state foaming process described in the Materials and Methods section. Fig. 6 shows characteristic porous structures obtained for the three types of materials, whereas Table 1 summarizes their main parameters.

A clear influence of both the $\mathrm{Zn}(\mathrm{OAc})_{2}$ and the $\mathrm{ZnO} N \mathrm{Ns}$ is found in the final porous structure of the foamed films. The PMMA/Zn(OAc $)_{2}$ films present an increase of the average pore size, from 4.35 to $14.42 \mu \mathrm{m}$, and a significant decrease of the pore nucleation density from $5.05 \times 10^{10}$ to $9.24 \times 10^{8}$ nuclei/ $/ \mathrm{cm}^{3}$ compared to the neat PMMA samples. On the other hand, the presence of the ZnO NPs is responsible for both the decrease of the average pore size of the PMMA-based films from 4.35 to $0.64 \mu \mathrm{m}$, and for the increase of the pore nucleation density by two orders of magnitude, from about $5.05 \times 10^{10}$ to $1.81 \times 10^{12}$ nuclei $/ \mathrm{cm}^{3}$. The results clearly demonstrate that the ZnO NPs improve the foaming behavior of the PMMA films by inducing a heterogeneous nucleation in the PMMA matrix, which allows decreasing the pore size of the foamed films below the micron. These results are supported by previous works, in which was established that the critical nucleation radius for the $\mathrm{PMMA} / \mathrm{CO}_{2}$ system for saturation pressures between 10 and $30 \mathrm{MPa}$ should present values between 0.2 and $8 \mathrm{~nm}$, and therefore, individual nanoparticles with sizes in that range, such as the ZnO NPs herein reported $(6 \mathrm{~nm})$, can induce the heterogeneous nucleation $[16,18]$.

It should be noted here that the pore nucleation density achieved with the ZnO NPs could be not enough to obtain low or medium density nanoporous foams [4]; however, it is expected that further enhancement of the production route, employed for the first time in this work for the production of polymer foams, will overcome this limitation.

\subsection{Templated PMMA-based films}

The clear differences found on the pore nucleation density between the PMMA/ZnO NPs and both PMMA and PMMA/Zn(OAc) should be sufficient to develop templated PMMA-based foams with variable pore sizes at different regions throughout their volume. For this reason, $\mathrm{PMMA} / \mathrm{Zn}(\mathrm{OAc})_{2}$ films were subjected to the heterogeneous thermal annealing described in the Materials and Methods section, with the aim to obtain a templated film with well-

Table 1

Main features of the porous structure of the foamed films: pore average size ( $\left.\varphi_{3 \mathrm{D}}\right)$, porosity $\left(\mathrm{V}_{\mathrm{f}}\right)$, and pore nucleation density $\left(\mathrm{N}_{\mathrm{o}}\right)$.

\begin{tabular}{llll}
\hline Samples & $\varphi_{3 \mathrm{D}}(\mu \mathrm{m})$ & $\mathrm{V}_{\mathrm{f}}$ & $\mathrm{N}_{\mathrm{o}}\left(\right.$ nuclei $\left./ \mathrm{cm}^{3}\right)$ \\
\hline PMMA & $4.35 \pm 1.21$ & $0.69 \pm 0.05$ & $5.05 \times 10^{10} \pm 3.5 \times 10^{9}$ \\
PMMA/Zn $(\mathrm{OAc})_{2}$ & $14.42 \pm 4.89$ & $0.59 \pm 0.05$ & $9.24 \times 10^{8} \pm 7.7 \times 10^{7}$ \\
PMMA/ZnO NPs & $0.64 \pm 0.38$ & $0.20 \pm 0.03$ & $1.81 \times 10^{12} \pm 2.7 \times 10^{11}$ \\
\hline
\end{tabular}

differentiated PMMA/ZnO NPs and PMMA/Zn(OAc $)_{2}$ regions. The developed setup for the heterogeneous thermal annealing successfully controlled the temperature in both regions, since after the $14 \mathrm{~h}$ annealing process it was proved by thermographic analysis that the heated region of the film presents a temperature about $100{ }^{\circ} \mathrm{C}$, whereas the other region remains at $0{ }^{\circ} \mathrm{C}$, with just a small transition area between both regions (see Fig. S2 in the Supplementary Information). Taking into account the thermographic results from several samples, as well as other experimental results described in the following sections, it was possible to identify the average length of each region. For a film of $30 \mathrm{~mm}$ long the annealed part at $100{ }^{\circ} \mathrm{C}$ was $12.5 \mathrm{~mm}$, followed by a $5 \mathrm{~mm}$ transition length, and finally the last $12.5 \mathrm{~mm}$ of the film were always kept at $0{ }^{\circ} \mathrm{C}$ (Fig. 7 ).

With the aim to prove the efficiency of the thermal treatment it was analysed by XRD the presence of $\mathrm{ZnO}$ and/or $\mathrm{Zn}(\mathrm{OAc})_{2}$ in both regions, as well as in the transition area, as shown in Fig. 7.

XRD results perfectly confirm the heterogeneous thermal annealing of the samples. The region kept at $0{ }^{\circ} \mathrm{C}$ only presents the peaks corresponding to the $\mathrm{Zn}(\mathrm{OAc})_{2}$, whereas the region annealed at $100{ }^{\circ} \mathrm{C}$ clearly shows only the peaks corresponding to the $\mathrm{ZnO}$ NPs (see Fig. 2 for comparison). Moreover, the transition area presents peaks of both $\mathrm{Zn}(\mathrm{OAc})_{2}$ and $\mathrm{ZnO}$ NPs. Therefore, it was demonstrated that using a simple heterogeneous thermal treatment it is possible to obtain templated PMMA-based films, with diverse compositions at different areas, where only a selected region presents homogeneously distributed ZnO NPs.

\subsection{Foaming of templated PMMA-based films}

Finally, the templated PMMA-based films were subjected to the same foaming procedure employed for the neat PMMA, PMMA/ $\mathrm{Zn}(\mathrm{OAc})_{2}$, and PMMA/ZnO NPs films (described in the Materials and Methods section). SEM micrographs of the resulting porous structures in the three distinct regions of the templated PMMA-based films are shown in Fig. 8.

Clear differences are found between the porous structure of the region annealed at $100{ }^{\circ} \mathrm{C}$ and the region kept at $0{ }^{\circ} \mathrm{C}$, with an increased number of smaller pores present in the first one, which is in good agreement with the presence of ZnO NPs nucleation agents in this region and their absence in the other. Moreover, the transition region seems to present an intermediate behavior, which corresponds to the partial conversion of $\mathrm{Zn}(\mathrm{OAc})_{2}$ into $\mathrm{ZnO}$ NPs (see Fig. 7). A more detailed analysis of the porous structure was carried out in 5 areas along the templated PMMA-based films (two in the annealed zone, one in the intermediate zone, and other two in the non-annealed zone), with the aim to ensure the presence of a different pore nucleation density between the annealed and not annealed regions.

Fig. 9 summarizes the main obtained results, pore size and pore nucleation density evolution, whereas Table 2 provides also the porosity of each region of the foamed templated film. 


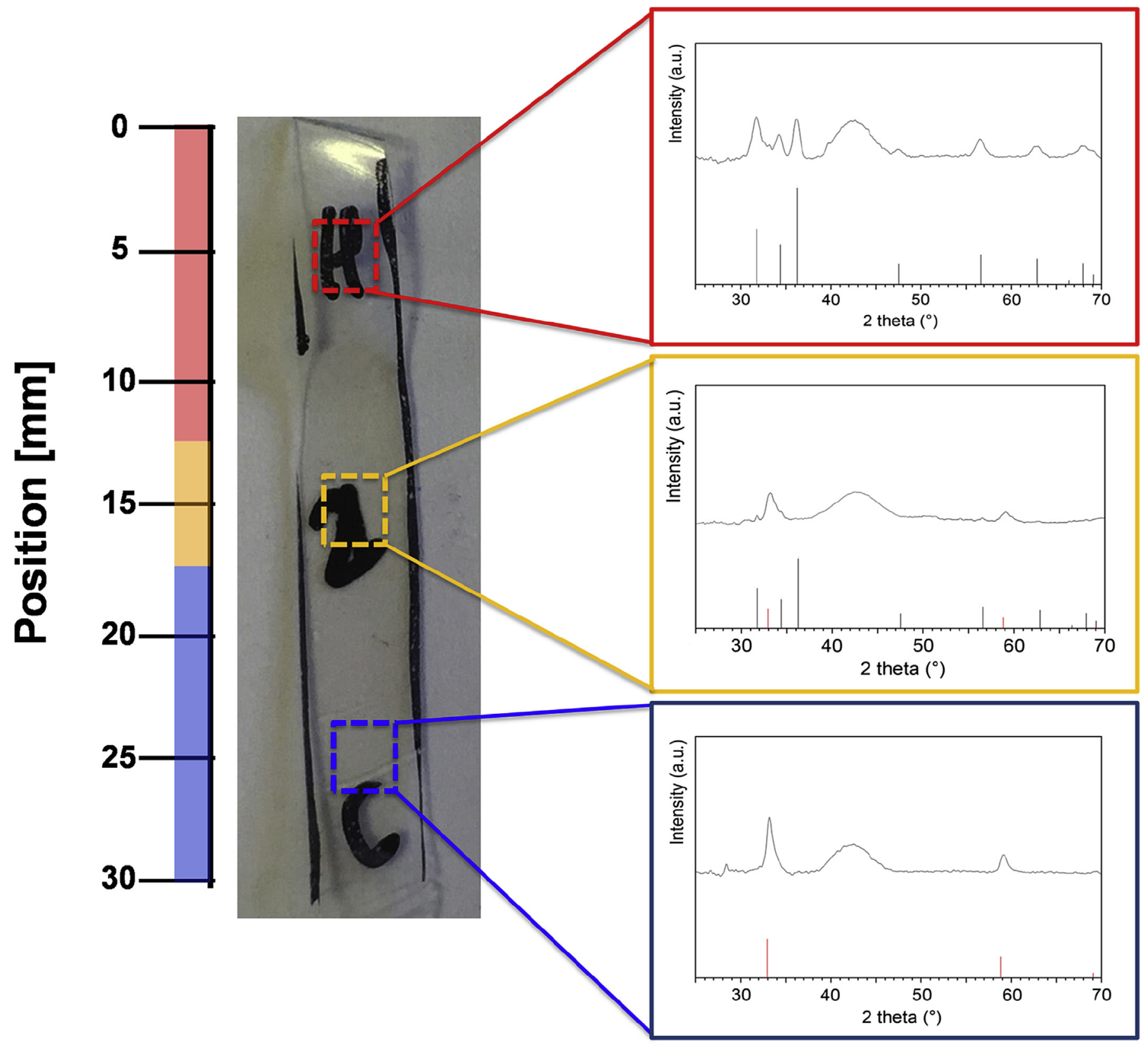

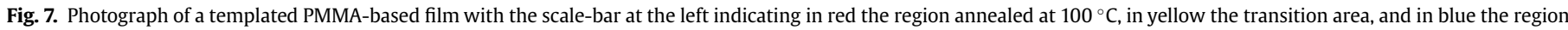

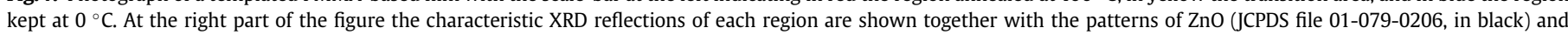
$\mathrm{Zn}(\mathrm{OAc})_{2}$ (JCPDS file 00-056-0569, in red). (For interpretation of the references to colour in this figure legend, the reader is referred to the web version of this article.)

First, it should be noticed that a different pore size is obtained at the regions with and without $\mathrm{ZnO}$ NPs, with the pore size increasing from about $500 \mathrm{~nm}$ to more than $1 \mu \mathrm{m}$ (Fig. 9). Moreover, the evolution of the pore nucleation density along the templated PMMA-based foamed samples (Fig. 9) shows a behavior which agrees with their production process. The region annealed at $100{ }^{\circ} \mathrm{C}$ (PMMA/ZnO NPs) shows a rather constant pore nucleation density about $2-3 \times 10^{12}$ nuclei $/ \mathrm{cm}^{3}$, in the same order of magnitude with the PMMA/ZnO NPs films (see Table 1). Then, the pore nucleation density starts to decrease in the transition area as a consequence of the incomplete conversion of PMMA/Zn(OAc $)_{2}$ into PMMA/ZnO NPs. Finally it reaches significantly lower values about $2-3 \times 10^{11}$ nuclei $/ \mathrm{cm}^{3}$ in the region that was kept at $0{ }^{\circ} \mathrm{C}$ (PMMA/Zn $\left.(\mathrm{OAc})_{2}\right)$.
Although two regions with clearly differentiated pore size and pore nucleation density were obtained throughout the templated PMMA-based foam, some differences between the templated and the homogeneous PMMA-based films should be discussed. It can be seen that the porosity of the templated PMMA-based foams is rather constant along the diverse regions (between 0.12 and 0.20 ), being in the same range with the PMMA/ZnO NPs foams $(0.20$, see Table 1). Thus, the PMMA/Zn(OAc $)_{2}$ region of the templated foams was not able to reach the same expansion with the homogeneous PMMA/Zn $(\mathrm{OAc})_{2}$ foams (porosity 0.59 , see Table 1 ), most likely due to the physical continuity of the templated sample (i.e. the expansion of the PMMA/Zn(OAc) $)_{2}$ region is constrained by the low expansion of the PMMA/ZnO NPs region). On the other hand, this rather constant porosity allows a direct comparison between the 


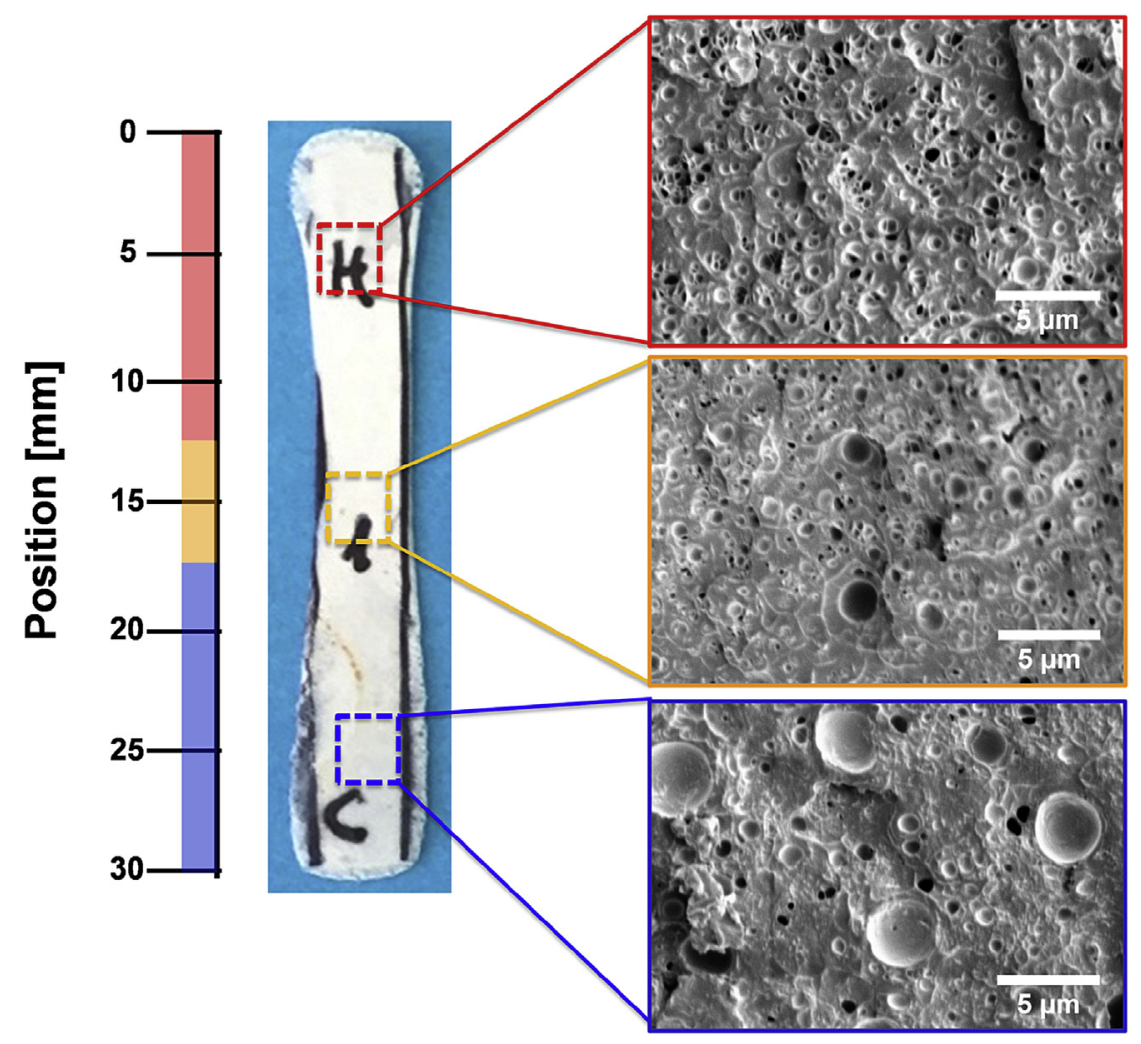

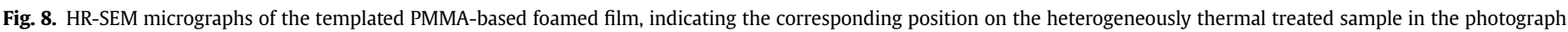

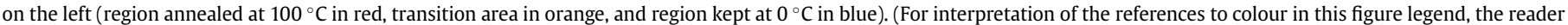
is referred to the web version of this article.)

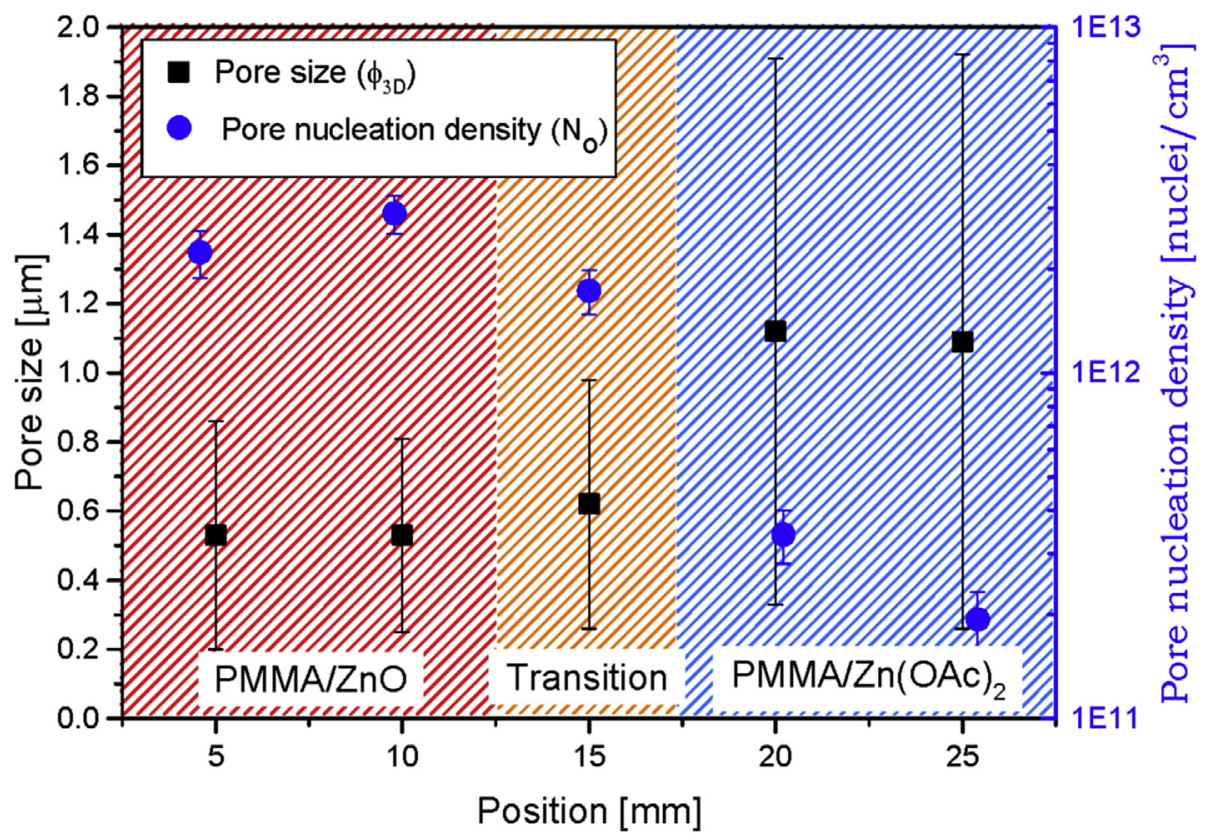

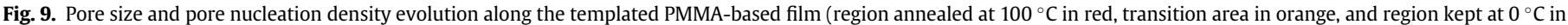
blue). (For interpretation of the references to colour in this figure legend, the reader is referred to the web version of this article.)

pore sizes of the diverse regions, ensuring that the pore size trend found is directly related to the pore nucleation mechanisms and not to different porosities of the foam. Moreover, there is a notable difference between the pore nucleation density of the PMMA/
$\mathrm{Zn}(\mathrm{OAc})_{2}$ region of the templated PMMA-based foams $\left(2-3 \times 10^{11}\right.$ nuclei $/ \mathrm{cm}^{3}$ ) and of the PMMA $/ \mathrm{Zn}(\mathrm{OAc})_{2}$ homogeneous foams $\left(9.24 \times 10^{8}\right.$ nuclei $\left./ \mathrm{cm}^{3}\right)$. It should be taken into account that the $\mathrm{PMMA} / \mathrm{Zn}(\mathrm{OAc})_{2}$ region of the templated foams could provide a 
Table 2

Main features of the porous structure of the templated foamed film (positions correspond to Figs. 6 and 7$)$ : pore average size $\left(\varphi_{3 \mathrm{D}}\right)$, porosity $\left(\mathrm{V}_{\mathrm{f}}\right)$, and pore nucleation density $\left(\mathrm{N}_{\mathrm{o}}\right)$.

\begin{tabular}{llll}
\hline Position $(\mathrm{mm})$ & $\varphi_{3 \mathrm{D}}(\mu \mathrm{m})$ & $\mathrm{V}_{\mathrm{f}}$ & $\mathrm{N}_{\mathrm{o}}\left(\right.$ nuclei $\left./ \mathrm{cm}^{3}\right)$ \\
\hline 5 & $0.53 \pm 0.33$ & $0.15 \pm 0.02$ & $2.23 \times 10^{12} \pm 3.5 \times 10^{11}$ \\
10 & $0.53 \pm 0.28$ & $0.18 \pm 0.02$ & $2.89 \times 10^{12} \pm 3.7 \times 10^{11}$ \\
15 & $0.62 \pm 0.36$ & $0.18 \pm 0.03$ & $1.73 \times 10^{12} \pm 2.5 \times 10^{11}$ \\
20 & $1.12 \pm 0.79$ & $0.20 \pm 0.03$ & $3.40 \times 10^{11} \pm 6.0 \times 10^{10}$ \\
25 & $1.09 \pm 0.83$ & $0.12 \pm 0.02$ & $1.93 \times 10^{11} \pm 3.9 \times 10^{10}$ \\
\hline
\end{tabular}

more accurate information about the pore nucleation, as it is expected that its low expansion freezes the pore evolution virtually just after the pore nucleation. On the contrary, the PMMA/Zn(OAc) homogeneous foams could present pore coalescence or/and coarsening during the pores' growth that prohibit the calculation of an accurate pore nucleation density, since this is obtained from the final pore density and porosity of the porous structure [67].

It is well-known that the simultaneous presence of pores with significant differences in size in the early stages of the pores' growth, such as the ones shown by the PMMA/Zn(OAc $)_{2}$ region of the templated foam, promotes the coarsening of the pores during the subsequent stages of the pores' growth (see Supplementary Information) [70]. This coarsening at the subsequent foaming stages could be possible only in the homogeneous PMMA/Zn(OAc) samples, whereas it was prohibited in the templated samples due to the restricted expansion of the PMMA/Zn(OAc $)_{2}$ area. The presence of few very big pores near the borders of the PMMA/Zn(OAc) 2 area of the templated foams are a further indication of pore coarsening that could not be realized in the central part of this area due to its low expansion (see Fig. S4 in the Supplementary Information). Therefore, the pore sizes found in the PMMA/Zn(OAc) foams, bigger than the ones of the PMMA foams (see Table 1), are mainly a consequence of the coarsening favored by the presence of the $\mathrm{Zn}(\mathrm{OAc})_{2}$.

Finally, it should be noticed that the design of the experiments (see Materials and Methods section), as well as the foaming mould (see Fig. S3), ensures that all the foaming parameters (e.g. temperature in each stage, gas diffusion in/out of the sample) are the same along the PMMA-based films. Therefore, the differences found in the pore nucleation densities cannot be related to other parameter than to the presence or absence of the ZnO NPs. Therefore, we can safely claim that the spatially selected in situ synthesis of nanoparticles is a suitable approach to obtain polymer foams with a tunable templated pore size. Moreover, it is expected that this approach can be further optimized, including also the use of laser radiation as initiator of the NPs synthesis [55] to obtain templated samples with a faster procedure and more precise and complex templates.

\section{Conclusions}

PMMA-based templated foams with controlled pore sizes have been obtained for the first time by gas dissolution foaming, taking advantage of the in situ synthesis of ZnO NPs into selected areas of the polymer matrix. First, it was demonstrated that it is possible to completely convert the $\mathrm{Zn}(\mathrm{OAc})_{2}$ precursor incorporated in the PMMA into crystalline ZnO NPs after $14 \mathrm{~h}$ of thermal treatment at $100{ }^{\circ} \mathrm{C}$. Using this method, ZnO NPs with well-controlled mean particles size of $6.5 \pm 1.2 \mathrm{~nm}$ have been homogeneously distributed in the polymer matrix. Secondly, it was proved that upon foaming process the previously formed ZnO NPs present a favorable effect on the pore nucleation inside the composite matrix. Indeed, the PMMA/ZnO NPs nanocomposite foams had pore sizes below the micron, whereas the neat PMMA foams showed pore sizes of $4 \mu \mathrm{m}$ under the same conditions. Finally, by a heterogeneous thermal treatment of PMMA/Zn(OAc $)_{2}$ samples were obtained templated heterogeneous PMMA-based films with varying distributions of ZnO NPs throughout the films. These templated heterogeneous PMMA composite samples allowed for the first time, using $\mathrm{CO}_{2}$ foaming, the production of templated foams, with welldifferentiated regions of tunable pore nucleation density and pore size, as a consequence of the presence of different concentrations of ZnO nanoparticles.

This new kind of process can allow the development of polymer foams with improved properties and an unusual templated porous structure, which can confer a superior performance in applications such as acoustic insulation or electromagnetic interference shielding, as well as provide the basis for the development of new functions.

\section{Acknowledgments}

Financial support from FPU grant FPU14/02050 (V. Bernardo) from the Spanish Ministry of Education is gratefully acknowledged. Financial assistance from MINECO, FEDER, UE (MAT2015-69234-R) and the Junta of Castile and Leon (VA011U16) are gratefully acknowledged. We also thank the organizers of the 2014 IIT Nanophysics Retreat for the fruitful discussions, which led to this work.

\section{Appendix A. Supplementary data}

Supplementary data related to this article can be found at http:// dx.doi.org/10.1016/j.polymer.2017.07.067.

\section{References}

[1] D. Klempner, V. Sendijarevi'c, R.M. Aseeva, Handbook of Polymeric Foams and Foam Technology, Hanser Publishers, Munich, Germany, 2004.

[2] T. Sadik, C. Pillon, C. Carrot, J.A. Reglero Ruiz, M. Vincent, N. Billon, J. Cell. Plast. (2016), http://dx.doi.org/10.1177/0021955x16633643.

[3] B. Notario, J. Pinto, E. Solorzano, J.A. de Saja, M. Dumon, M.A. Rodríguez-Pérez, Polymer 56 (2015) 57-67.

[4] S. Costeux, J. Appl. Polym. Sci. (2014) 131.

[5] B. Notario, J. Pinto, M.A. Rodriguez-Perez, Prog. Mater Sci. 78-79 (2016) 93-139.

[6] D. Miller, V. Kumar, Polymer 52 (2011) 2910-2919.

[7] B. Notario, J. Pinto, M.A. Rodríguez-Pérez, Polymer 63 (2015) 116-126.

[8] C. Forest, P. Chaumont, P. Cassagnau, B. Swoboda, P. Sonntag, Prog. Polym. Sci. 41 (2015) 122-145

[9] S. Liu, J. Duvigneau, G.J. Vancso, Eur. Polym. J. 65 (2015) 33-45.

[10] B. Notario, J. Pinto, R. Verdejo, M.A. Rodríguez-Pérez, Polymer (2016), http:// dx.doi.org/10.1016/j.polymer.2016.11.030.

[11] B. Notario, A. Ballesteros, J. Pinto, M.A. Rodríguez-Pérez, Mat. Lett. 168 (2016) 76-79.

[12] J. Martín-de León, V. Bernardo, M. Rodríguez-Pérez, Polymers 8 (2016) 265.

[13] J. Pinto, B. Notario, R. Verdejo, M. Dumon, S. Costeux, M.A. Rodriguez-Perez, Polymer 113 (2017) 27-33.

[14] X. Lu, R. Caps, J. Fricke, C.T. Alviso, R.W. Pekala, J. Non-Cryst. Solids 188 (1995) 226-234.

[15] C. Forest, P. Chaumont, P. Cassagnau, B. Swoboda, P. Sonntag, Polymer 58 (2015) 76-87.

[16] S. Costeux, L. Zhu, Polymer 54 (2013) 2785-2795.

[17] J.A. Reglero Ruiz, M. Dumon, J. Pinto, M.A. Rodriguez-Pérez, Macromol. Mat. Eng. 296 (2011) 752-759.

[18] J. Pinto, M. Dumon, M. Pedros, J. Reglero, M.A. Rodriguez-Perez, Chem. Eng. J. 243 (2014) 428-435.

[19] S. Liu, B. Zoetebier, L. Hulsman, Y. Zhang, J. Duvigneau, G.J. Vancso, Polymer 104 (2016) 22-30.

[20] V. Bernardo, J. Martín-de León, M.A. Rodríguez-Pérez, Mat. Lett. 178 (2016) $155-158$.

[21] S. Costeux, P. Bunker, H.K. Jeon, J. Mat. Res. 28 (2013) 2351-2365.

[22] L. Li, T. Nemoto, K. Sugiyama, H. Yokoyama, Macromolecules 39 (2006) $4746-4755$

[23] S. Siripurapu, J.M. DeSimone, S.A. Khan, R.J. Spontak, Macromolecules 38 (2005) 2271-2280.

[24] L. Li, H. Yokoyama, T. Nemoto, K. Sugiyama, Adv. Mat. 16 (2004) 1226-1229. 
[25] J.A.R. Ruiz, M. Pedros, J.-M. Tallon, M. Dumon, J. Supercrit. Fluids 58 (2011) $168-176$.

[26] D. Miller, P. Chatchaisucha, V. Kumar, Polymer 50 (2009) 5576-5584.

[27] C. Dutriez, K. Satoh, M. Kamigaito, H. Yokoyama, RSC Adv. 2 (2012) 2821.

[28] T. Otsuka, K. Taki, M. Ohshima, Macromol. Mat. Eng. 293 (2008) 78-82.

[29] T. Nemoto, J. Takagi, M. Ohshima, Polym. Eng. Sci. 50 (2010) 2408-2416.

[30] H. Yu, Y. Lei, X. Yu, X. Wang, T. Liu, S. Luo, J. Polym. Res. (2016) 23.

[31] H. Guo, V. Kumar, Polymer 56 (2015) 46-56.

[32] T.J. Yoon, W. Kong, D.E. Kwon, B.K. Park, W.I. Lee, Y.-W. Lee, J. Supercrit. Fluids (2017), http://dx.doi.org/10.1016/j.supflu.2017.01.004.

[33] H. Guo, A. Nicolae, V. Kumar, J. Polym. Sci. Part B Polym. Phys. 53 (2015) 975-985.

[34] H. Guo, V. Kumar, Polymer 57 (2015) 157-163.

[35] H. Guo, A. Nicolae, V. Kumar, Polymer 70 (2015) 231-241.

[36] P. Spitael, C.W. Macosko, R.B. McClurg, Macromolecules 37 (2004) 6874-6882.

[37] B. Krause, K. Diekmann, N.F.A.V.D. Vegt, M. Wessling, Macromolecules 35 (2002) 1738-1745.

[38] R. Zhang, C. Dutriez, K. Sugiyama, T. Ishizone, H. Yokoyama, Soft Matter 7 (2011) 4032.

[39] T. Shinkai, M. Ito, K. Sugiyama, K. Ito, H. Yokoyama, Soft Matter 8 (2012) 5811.

[40] S.N. Leung, A. Wong, Q. Guo, C.B. Park, J.H. Zong, Chem. Eng. Sci. 64 (2009) 4899-4907.

[41] Y. Kim, C.B. Park, P. Chen, R.B. Thompson, Polymer 52 (2011) 5622-5629.

[42] X. Xu, D.E. Cristancho, S. Costeux, Z.-G. Wang, Soft Matter 9 (2013) 9675.

[43] A. Saint-Jalmes, Soft Matter 2 (2006) 836.

[44] J. Pinto, J.A. Reglero-Ruiz, M. Dumon, M.A. Rodriguez-Perez, J. Supercrit. Fluids 94 (2014) 198-205.

[45] S.N. Leung, A. Wong, C.B. Park, J.H. Zong, J. Appl. Polym. Sci. 108 (2008) 3997-4003.

[46] J. Pinto, M. Dumon, M.A. Rodriguez-Perez, R. Garcia, C. Dietz, J. Phys. Chem. C 118 (2014) 4656-4663.

[47] S. Pardo-Alonso, E. Solórzano, S. Estravís, M.A. Rodríguez-Perez, J.A. de Saja, Soft Matter 8 (2012) 11262.

[48] J. Dlouhá, L. Suryanegara, H. Yano, Soft Matter 8 (2012) 8704.

[49] Y.H. Lee, K.H. Wang, C.B. Park, M. Sain, J. Appl. Polym. Sci. 103 (2007) 2129-2134.

[50] D. Morselli, F. Bondioli, M. Sangermano, M. Messori, Polym. Eng. Sci. 55 (2014)
1689-1697.

[51] L.X. Reynolds, T. Lutz, S. Dowland, A. MacLachlan, S. King, S.A. Haque, Nanoscale 4 (2012) 1561-1564.

[52] A.M. Laera, V. Resta, E. Piscopiello, V. Miceli, M. Schioppa, A.G. Scalone, F.D. Benedetto, L. Tapfer, Nanoscale Res. Lett. 8 (2013) 382.

[53] F. Spano, A. Massaro, L. Blasi, M. Malerba, R. Cingolani, A. Athanassiou, Langmuir 28 (2012) 3911-3917.

[54] M. Mehrabanian, D. Fragouli, D. Morselli, A. Scarpellini, G.C. Anyfantis, A. Athanassiou, Mat. Res. Express 2 (2015) 105014.

[55] D. Morselli, A. Scarpellini, A. Athanassiou, D. Fragouli, RSC Adv. 6 (2016) 11412.

[56] A. Athanassiou, L. Blasi, M. De Giorgi, G. Caputo, D. Fragouli, E. Tsiranidou, A.M. Laera, L. Tapfer, R. Cingolani, Polym. Compos. (2009) 1075-1083, http:// dx.doi.org/10.1002/pc.20894.

[57] D. Fragouli, P.P. Pompa, M. Kalyva, G. Caputo, L. Tapfer, R. Cingolani, A. Athanassiou, J. Phys. Chem. C 114 (2010) 13985-13990.

[58] D. Fragouli, V. Resta, P.P. Pompa, A.M. Laera, G. Caputo, L. Tapfer, R. Cingolani, A. Athanassiou, Nanotechnology 20 (2009) 155302.

[59] A. Athanassiou, R. Cingolani, E. Tsiranidou, C. Fotakis, A.M. Laera, E. Piscopiello, L. Tapfer, Appl. Phys. Lett. 91 (2007) 153108.

[60] W.J.E. Beek, M.M. Wienk, R.A.J. Janssen, Adv. Mat. 16 (2004) 1009-1013.

[61] W.J.E. Beek, L.H. Slooff, M.M. Wienk, J.M. Kroon, R.A.J. Janssen, Adv. Funct. Mat. 15 (2005) 1703-1707.

[62] S. Ghaffari Mosanenzadeh, H.E. Naguib, C.B. Park, N. Atalla, J. Mat. Sci. 50 (2014) 1248-1256.

[63] L. Monnereau, L. Urbanczyk, J.-M. Thomassin, T. Pardoen, C. Bailly, I. Huynen, C. Jérôme, C. Detrembleur, Polymer 59 (2015) 117-123.

[64] J. Yu, L. Song, F. Chen, P. Fan, L. Sun, M. Zhong, J. Yang, Mat. Today Commun. 9 (2016) $1-6$.

[65] M.T. Ngo, J.S. Dickmann, J.C. Hassler, E. Kiran, J. Supercrit. Fluids 109 (2016) $1-19$.

[66] M.D. Abramoff, P.J. Magalhães, S.J. Ram, Biophot. Inter. 11 (2004) 36-42.

[67] J. Pinto, E. Solorzano, M.A. Rodriguez-Perez, J.A. de Saja, J. Cell. Plast. 49 (2013) $555-575$.

[68] E.A. Meulenkamp, J. Phys. Chem. B 102 (1998) 5566-5572.

[69] D.W. Bahnemann, C. Kormann, M.R. Hoffmann, J. Phys. Chem. 91 (1987) 3789-3798.

[70] Z. Zhu, D. Xu, C.B. Park, R.G. Fenton, J. Cell. Plast. 41 (2005) 475-486. 